\title{
Review
}

\section{What is Functional Mobility Applied to Parkinson's Disease?}

\author{
Raquel Bouça-Machado ${ }^{\mathrm{a}, \mathrm{b}}$, Walter Maetzler ${ }^{\mathrm{c}}$ and Joaquim J. Ferreira ${ }^{\mathrm{a}, \mathrm{b}, \mathrm{d}, *}$ \\ ${ }^{a}$ Instituto de Medicina Molecular, Lisbon, Portugal \\ ${ }^{\mathrm{b}}$ CNS-Campus Neurológico Sénior, Torres Vedras, Portugal \\ ${ }^{\mathrm{c}}$ Department of Neurology, Christian-Albrechts-University of Kiel, Kiel, Germany \\ ${ }^{\mathrm{d}}$ Laboratory of Clinical Pharmacology and Therapeutics, Faculty of Medicine, University of Lisbon, Portugal
}

\begin{abstract}
Although yet poorly defined and often misused, the concept of functional mobility has been used in research studies as a more global and ecological outcome of patients' health status. Functional mobility is a person's physiological ability to move independently and safely in a variety of environments in order to accomplish functional activities or tasks and to participate in the activities of daily living, at home, work and in the community. Parkinson's disease (PD) has a direct impact on patients' motor control and on mobility in general. Even with optimal medical management, the progression of PD is associated with mounting impairments at different levels of body function, causing marked limitations in a wide variety of activities, as well as a severe disability and loss of autonomy. Despite this, for everyday functioning PD patients need to have a good functional mobility that allow them to get around effortlessly in a reasonable amount of time to access to the same environments as others. This paper reviewed the concept of functional mobility applied to PD. This was done through an International Classification of Functioning and Disability (ICF) perspective. Recommendations to address the known factors that contribute to a poor functional mobility were outlined while suggestions for clinical practice and research were made.
\end{abstract}

Keywords: Functional mobility, international classification of functioning, disability and health, Parkinson's disease

\section{INTRODUCTION}

What is functional mobility? Is there a difference in the functional mobility of two Parkinson's disease (PD) patients with similar gait disturbance, one using an assistive mobility device, the other not? How do health professionals account for these differences? This paper reviewed the functional mobility concept and its implications for PD patients' everyday functioning. It followed the International Classification of Functioning, Disability and Health (ICF) model. The ICF model goes beyond the usual focus on a

\footnotetext{
*Correspondence to: Joaquim J. Ferreira, Laboratorio de Farmacologia Clínica e Terapêutica, Faculdade de Medicina de Lisboa, Av. Prof. Egas Moniz, 1649-028 Lisboa, Portugal. Tel.: +351 21 7802120; E-mail: joaquimjferreira@gmail.com.
}

diagnosis, incorporating detailed information on how functional, societal aspects, and contextual factors contribute to a patient's health condition. Therefore, it allows to better understand and describe health and health-related problems and to improve communication between patients, health professionals, researchers, and policy makers $[1,2]$. This model have been previously used for studying PD patients' disability [3-5] and quality-of-life [6].

A PubMed search, from inception to June 2017, was made using the following search terms were: "Functional mobility", "Mobility", "Disability", "Participation restrictions" and "Parkinson's disease". Language and publication restrictions were not applied. Being a narrative review a systematic selection of the included studies was also not 
performed. In order to fully address the opening question, the concept of functional mobility was introduced and, through the ICF model, the factors related with PD body functions impairments and activity limitations that could affect functional mobility were presented. It was also discussed how functional mobility limitation may restrict patients' everyday functioning and the potential impact of contextual factors. Additionally, in the end of the review, the most suitable outcome tools and interventions to address PD functional mobility limitations were appraised.

\section{FUNCTIONAL MOBILITY}

Functional mobility is increasingly used as an outcome in clinical studies as it may provide a more global and functional perspective of patients' health conditions. However, it is still a poorly defined concept, being commonly equated with mobility or functionality (Fig. 1). According to Forhan \& Gill in a review on obesity [7], functional mobility is the physiological ability of people to move independently and safely in a variety of environments in order to accomplish functional activities or tasks and to participate in activities of daily living (ADL), at home, work and in the community. It includes movements like standing, bending, walking and climbing, which are the building blocks of ADL, and hence crucial to an individual's independent living and global health status [7-11]. Impaired functional mobility has been found to be associated with a greater risk of falls, loss of independence, and institutionalization $[4,10]$.

\section{INTERNATIONAL CLASSIFICATION OF FUNCTIONING AND DISABILITY (ICF)}

As formulated by the World Health Organization (WHO) in 2001, the ICF is conceptualized as a universal framework focused on the description of how people live with a health condition (Fig. 2) [4, 7, 12]. Three levels of human functioning are classified: 1) body functions and structures as physiological and psychological functions, as well as body impairments, and anatomical deficiencies; 2) limitations in performing tasks or actions; and 3) participation restrictions in daily-life. Contextual factors can be either personal, such as age, gender, experiences, and interests; or environmental like physical, social, and attitudinal environment. This model assumes that all levels of human functioning and contextual factors are interconnected, i.e., impairments in body functions and structures may induce problems in activities that leads to participation restrictions, which can be facilitated or hindered by environmental or personal factors $[1,3]$.

\section{PARKINSON'S DISEASE}

PD is the second most common neurodegenerative disease $[3,13,14]$. It is characterized by its motor (bradykinesia, associated with rest tremor and rigidity) and non-motor problems [1, 15, 16].

Despite the variety of therapeutic options, disease progression usually leads to impairments at different levels of body function, limitations in a wide variety of ADL, and in severe disability, social
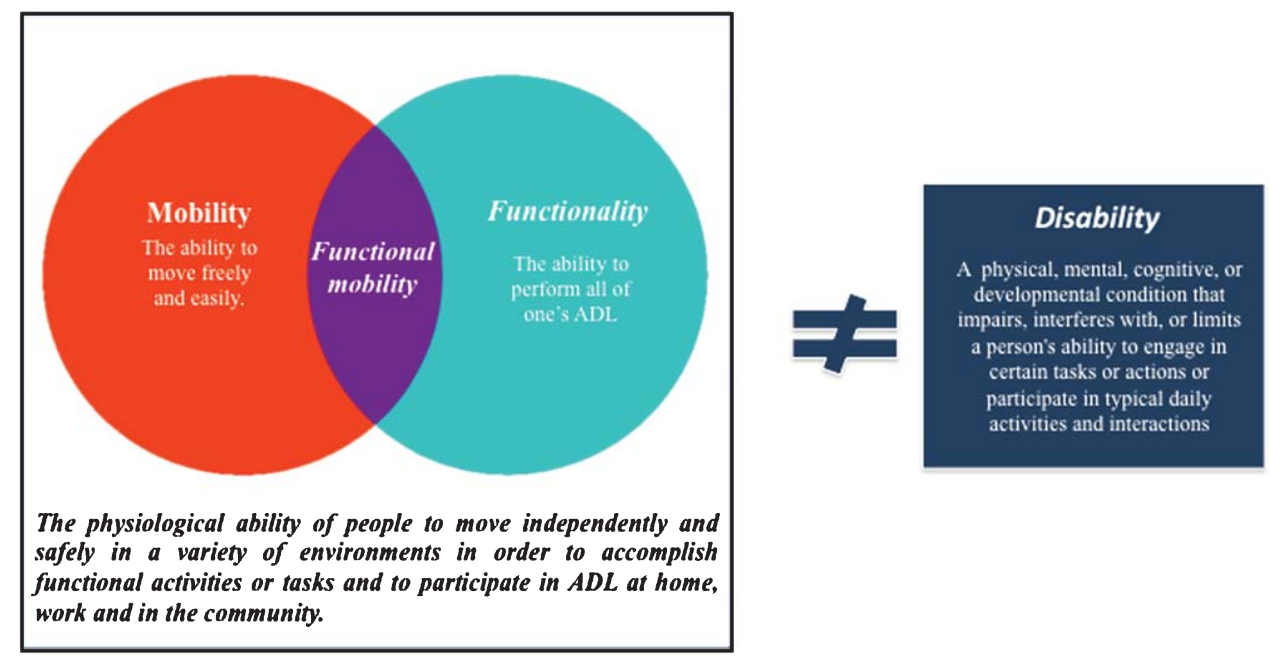

Fig. 1. Functional mobility concept. 


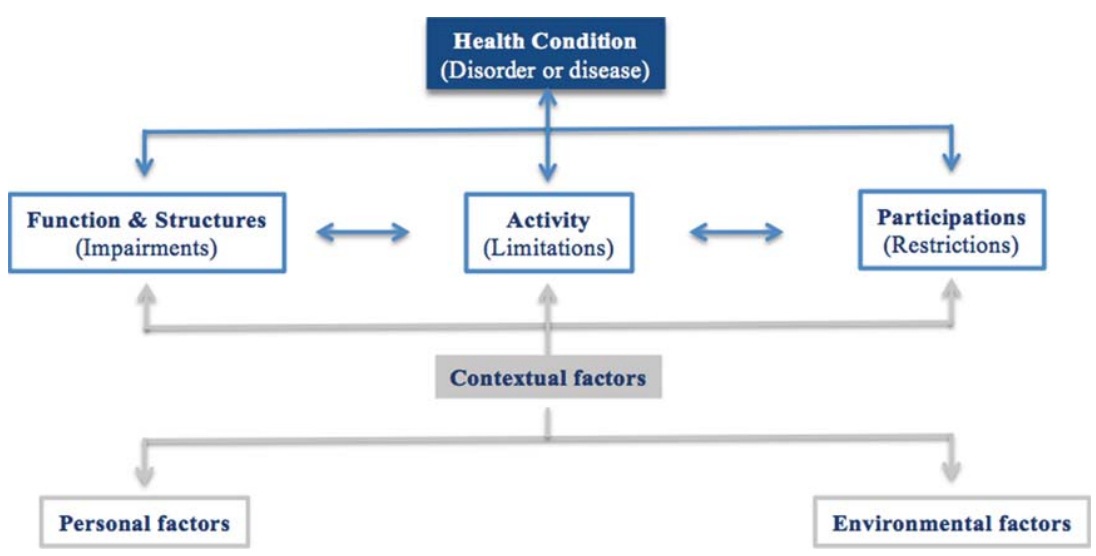

Fig. 2. ICF framework. Adapted from World Health Organization (2002) [12].

embarrassment and increasing dependence. Gradually, it reduces health-related quality of life (HrQoL) and increases the burden of patients and caregivers $[3,5,17,18]$.

\section{FUNCTIONAL MOBILITY IN PD: ICF-BASED METHODOLOGY}

In order to improve patients' global health status and reduce disease burden associated with functional immobility, it is important to understand a patient's personal needs, activity and environment [4]. In this section, we present the three levels of human functioning included in the ICF framework: 1) the impairments to body structures and functions relevant to PD patients' functional mobility; 2) how the activities that compose functional mobility are compromised by these impairments, in a functional perspective; 3) participation restrictions that PD patients may encountered, induced by functional mobility limitations; 4) lastly, some examples of frequent personal and environmental factors that influence the first three domains are presented (Figs. 3 and 4).

\section{Body functions and structures domain}

Functional mobility requires dynamic neural control to quickly and effectively adapt locomotion, balance, and postural transitions to changing environmental and task conditions. This in turn requires sensorimotor agility that involves: 1) coordination of complex sequences of movements, 2) on-going evaluation of environmental cues and contexts, 3) the ability to quickly switch motor programs with environmental changes, and 4) the ability to maintain safe mobility during multiple motor and cognitive tasks [7, 11].

\section{Motor symptoms}

Motor symptoms may contribute to functional mobility impairments directly, through gait impairments cause by non-dopaminergic pathways degeneration and indirectly due to bradykinesia and rigidity, which affect PD patients gait, balance and transitions [14, 19-21]. Gait impairments are complex to characterize because of the difficulty in distinguishing between the specific contribution of sensory, motor, and cognitive deficits and other factors like fear, muscle weakness or misjudgement of hazard risk. Evidence suggests that in later stage cholinergic dysfunction in the pedunculopontine nucleus has a key role in gait disturbance [14].

With disease progression, severe and disabling postural deformities are usually present (e.g., camptocormia, antecollis, pisa syndrome or scoliosis). These interfere with daily living activities, often leading to falls. Although still not well understood, a series of central and peripheral causes have been proposed to explain the complex and multifaceted underlying pathophysiology of these deformities $[14,22]$.

\section{Non-motor symptoms}

Functional mobility is also affected by PD nonmotor symptoms.

The inability to simultaneously carry out a cognitive and a motor task is a predictor of falls and a critical element to functional mobility. This has been found to be more difficult for PD patients than 


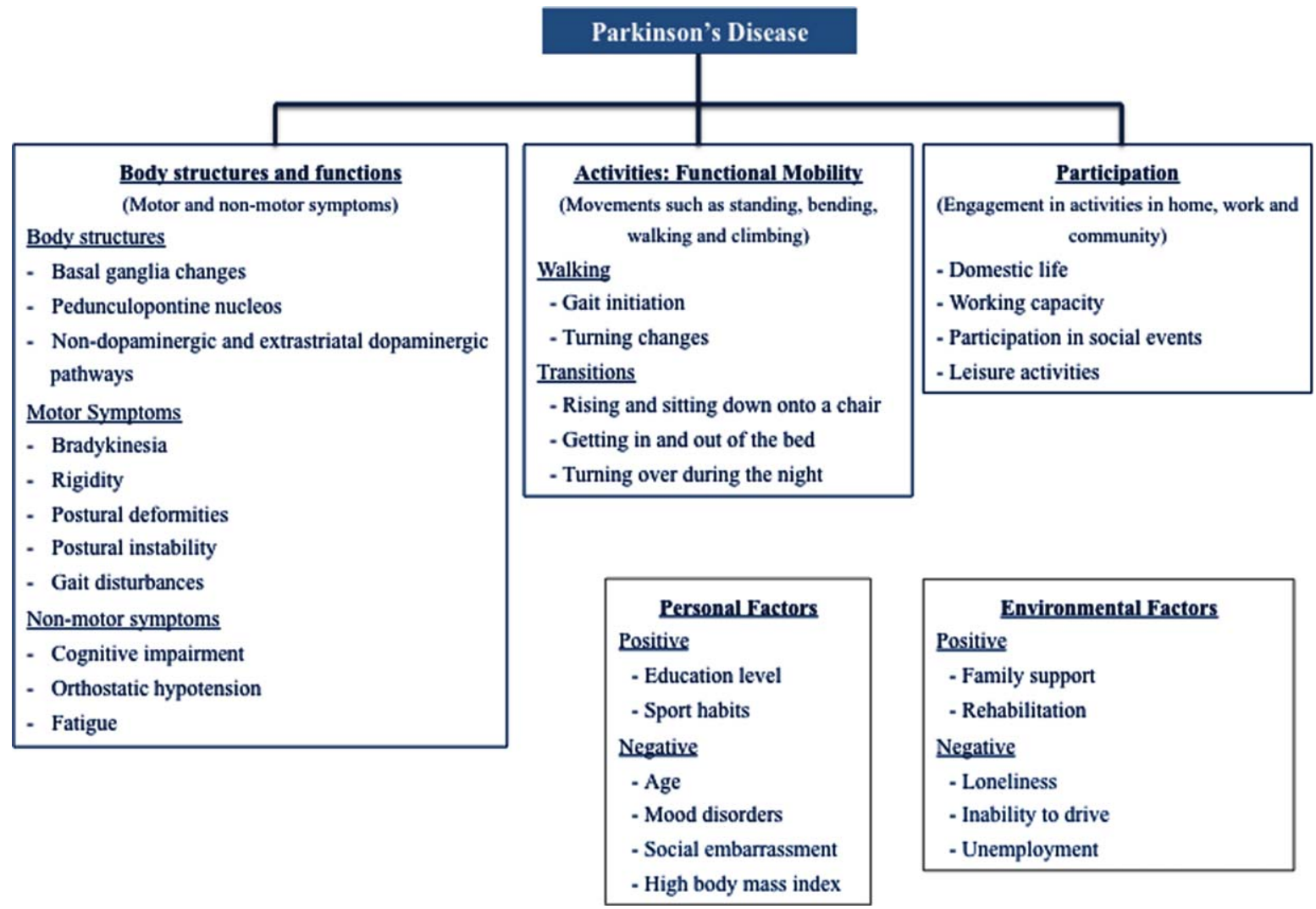

Fig. 3. The concept of functional mobility applied to PD in an ICF perspective.
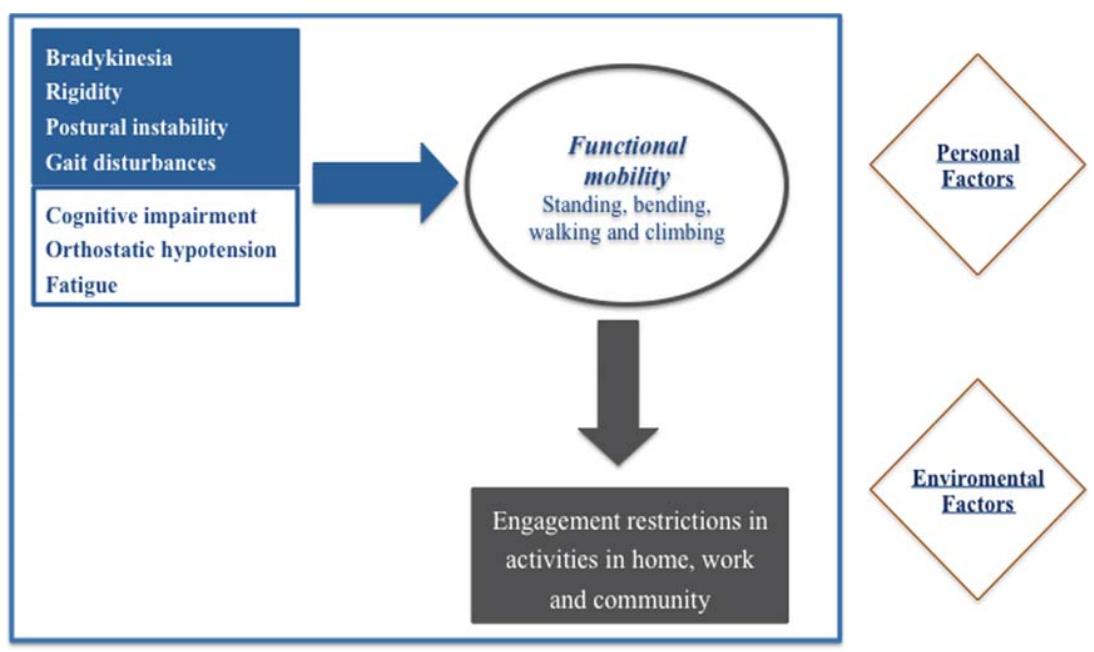

Fig. 4. Human domains and contextual factors contribution to PD functional mobility.

healthy controls, especially when walking is one of the tasks [11].

Dysautonomia seems also to play an important role in PD functional mobility. In concrete, orthostatic hypotension symptoms are a frequent complaint, associated to a higher prevalence of falls and a more rapid PD progression. It also affects mobility in general, patients' confidence in their own abilities and may undermine an active style of life [23]. 
Additionally, patients have frequently fatigue complaints, which has physical and psychological repercussions in PD functional mobility. The feeling of being tired all day and of not knowing how to get through the day makes fatigue, in patients perspective one of the symptoms most difficult to cope with $[14,24]$.

\section{Activity domain}

In $\mathrm{PD}$, activity limitations range from minor difficulties (e.g., fine motor coordination tasks) to more serious problems (e.g., skilled ADL motor tasks). Patients generally experience a loss of functional mobility resulting from the neurodegenerative effects of the disease in posture, balance, postural stability, and gait. Loss of independence in performing activities arises in the transition between Hoehn and Yahr (H\&Y) stages III and IV, and activities such as walking, housework, dressing and transfers are the most affected [1, 5, 7, 9, $13,25,26]$.

\section{Walking}

Patients describe gait disorders as a loss of confidence in walking, a feeling of imbalance or reduced ability to negotiate uneven terrain or stairs. A slower walking speed is often the first noticeable sign of parkinsonism [19].

Gait is defined as the forward propulsion of the body with rhythmical coordination of all four limbs combined with control of dynamic equilibrium of the body's center of mass. It is also a complex sensorimotor activity that involves spatial-temporal coordination of the legs, trunk and arms, as well as dynamic equilibrium.

Gait of PD patients have been shown to be: 1) significantly slower (typically $40-60 \mathrm{~m} / \mathrm{min}$ rather than $75-90 /$ min in age-matched controls), 2) with less foot clearance (foot's height during the swing phase) and an increased double phase support in the gait cycle (from the usual $20-30 \%$ of the gait cycle to over $35 \%), 3$ ) with smaller step lengths $(0.4-0.9 \mathrm{~m}$ for PD patients after withdrawal of medication or $0.8-1.0 \mathrm{~m}$ for those at the end-of-dose compared with $1.2-1.5 \mathrm{~m}$ for healthy older people), 4) narrow based, 5) asymmetrically reduced or absent arm swing 6) and stooped posture. Small shuffling steps (resulting from the reduced ground clearance and increased double phase support in gait cycle), a bilaterally reduced arm swing and slow, en bloc turns are also common [1, $14,27,28]$.
Walking problems are usually more pronounced during gait initiation, turning, walking through doorways and when performing simultaneous motor or cognitive tasks. These relates with the triggering of festination and freezing episodes, characterize by the sudden inability to generate effective stepping movements [28]. During festination episodes, the feet are behind the center of gravity, which causes rapid small steps. Freezing episodes are described by patients as having the feet "glued to the floor", which usually does not present as complete akinesia, but rather as shuffling with small steps or trembling of the legs.

\section{Transitions}

Throughout the course of disease, transitions become truly affected and predict risk of falls. Are particularly problematic: rising from, and sitting down on a chair, getting in or out and turning over in bed $[1,27]$. Sitting-to-standing is a complex component of some everyday functional tasks that requires the body to accelerate forward and then upward, and to transfer from a large to a small base of support to achieve an uprightb stance [1, 29]. PD patients exhibit a general slowness when compared to control subjects in performing this tasks with a spatiotemporal pattern preserved [30]. This indicates that PD patients' problems are not related with the selection, but in initiating and sequencing the appropriate motor program. Additionally, task analysis has shown that PD patients take a significantly longer time to complete each individual phase and a have a significantly smaller peak hip extension and ankle dorsiflexion torque when compared with control subjects [29]. The likely responsible factors are weak limb support against gravity (particularly reduced muscle power of the hip extensors), the difficulty in muscle activation and the inability to counteract unexpected external forces, vestibular impairment, and orthostatic hypotension [1, 29, 31]. PD patients seem also to have less body position changes during the night compared to the general population, which may affect sleep quality. Impaired bed mobility is often attributed to nocturnal hypokinesia, yet pain and overall muscle weakness and external factors such as bedcovers or reduced levels of levodopa at night, may also contribute to difficulty turning over in bed. The precise causal mechanism is still not clear $[1,32]$.

\section{Participation domain}

Participation problems are aspects of life as a member of society hindered by activity limitations [11]. Impairments in PD patients' functional mobility, may 
compromise involvement in leisure, work or social aspects of life in both household and community settings.

Working capacity, often affected in PD patients, is a concrete example of an important participation restriction related with functional mobility, not only because of work role in active fighting against exclusion from social and occupational environments, but also as livelihood $[7,33]$.

\section{Contextual factors}

Contextual factor could be personal or environmental, and have a positive or negative effect.

Age, a high body mass index, feeling disabled and social embarrassed represent some examples of personal factors with potential negative influence on PD patients' functional mobility. In contrast, high education levels and sport habits are examples of factors with a positive influence $[1,7,18]$.

Similarly, unemployment, loneliness and the inability to drive, are examples of possible environmental negative factors. The existence of family caregivers is the most valued environmental positive factor, once PD patients rely on them for most of their ADL needs $[1,7,18]$.

Within personal factors, perceived control (i.e., the person's belief of controlling the situations and act in accordance to that) is a prime candidate and a powerful predictor of active life and functional mobility [26]. PD clearly affects patients' perceived control, not only because of the impact of motor and non-motor symptoms on daily functional mobility, but also because of the unpredictability and social embarrassment frequently associated. This has multiple manifestations in patients' life, such as: to avoid walking on the street or in less familiar places due to fear of falling, concerns scheduling appointments because of not being sure of being able to get through it or to stay away from public places or social events to prevent feeling embarrassed with disease limitations $[2,16,19,26,34,35]$.

\section{FUNCTIONAL MOBILITY: SCALES AND TOOLS AVAILABLE}

Functional mobility is a global disease-related feature that may provide adequate information about treatment responses and disease course, as it may encompasses one of the outcomes most relevant to patients' daily lives [9].
Due to the heterogeneity and complexity of PD, its fluctuating nature and unpredictable medication response in advanced disease stages, clinical assessment is challenging and requires continuous prolonged periods of evaluation to reach an accurate picture of symptoms and their fluctuations [36].

The majority of PD studies that have measured functional mobility used rating scales like the MDS-UPDRS, infrequent events (e.g., falls) or subjective reports (e.g., diaries or questionnaires). Objective assessments, including the five-time sit-tostand (FTSTS) test and the timed up-and-go (TUG) test, are two of the most commonly used tools [10, 37, 38]. In 2015, Parashos and colleagues validated the "Ambulatory Capacity Measure". This is a measure of functional capacity, previous used in clinical trials, derived from UPDRS items related to falls, freezing, walking, gait and postural instability. It showed to be a good instrument, highly correlated with some of the most used outcome tool to assess functional capacity [39]. However, there is still no consensus about which screening tools are preferred or which outcomes are most suitable for monitoring functional mobility [40].

With technological advances, numerous devices have been created not only with the capacity of reliably evaluating fluctuating or rare events (e.g., freezing of gait or falls) that usually occur outside clinical visits, but also for obtaining more global, objective, and sensible outcomes for assessing patients' performance in ADL [41]. Yet, is still lacking to establish a specific protocol or metrics to measure PD-sensitive and specific functional mobility behaviours [27].

\section{IMPROVING FUNCTIONAL MOBILITY IN PD}

Due to PD heterogeneity, patients' experience of mobility impairment and respective coping strategies are very personal. In order to find an effective option is crucial to understand the patients' needs and offer suggestions according to local offerings, personal preferences, and cultural background $[9,11]$.

\section{Exercise programs}

Evidence shows that critical aspects of PD patients' functional mobility impairments (e.g., postural instability) are unresponsive to pharmacological and surgical therapies, making physical therapy an attractive option $[9,11]$. 
Previous animal studies have demonstrated that intense exercise programs can increase dopamine synthesis and release and improve brain function. Aerobic exercise (e.g., treadmill training) has shown to improve gait parameters, quality of life, and levodopa efficacy in PD patients. However, once functional mobility also depends on other components such as dynamic balance, dual tasking, and other sensorimotor skills, aerobic training is not sufficient to improve functional mobility in PD [11]. Task-specific exercises targeting a single, specific balance or gait impairment, in PD patients have also been tested with positive results [9].

Rehabilitation programs have been reported to be effective in preventing and improving PD patients' functional mobility when focusing on aerobic exercises and self-initiated movements, big and quick movements, large and flexible centers of mass control, reciprocal and coordinated movements of arms and legs, and rotational movements of torso over pelvis and pelvis over legs [11].

\section{Strategy training}

Strategy training is one of the key elements of physiotherapy PD management. It is defined as teaching the person how to move more easily and to maintain postural stability by using cognitive strategies. This includes two different methods: acquiring new motor skills (learning strategies) and compensating for movement disorders by bypassing the defective basal ganglia (compensating strategies) [42].

There is growing evidence that, at least in early PD, the capacity to learn new motor skills is not affected [42]. One study showed that PD patients with mean disease duration of 7 years have the capacity to learn new upper-limb movement sequences, improve performance and retain it for 48 hours [42, 43]. Another study evaluated a multiple-task gait-training program in mild PD patients (H\&Y stages II-III), reporting that study participants could maintain their learned increased multiple-task walking speed over 3 weeks $[42,44]$.

Compensatory strategies have been shown to be effective in moderate to severe PD patients, however requiring high mental effort and with relatively shortterm effects. They include: the use of visual (e.g., white lines on the floor) and auditory (e.g., rhythmical beat provided by a metronome) external cues, the visualization of walking with long steps, mental rehearsal of the desired movement pattern before performing the action and breaking down long or complex motor sequences into parts and focusing on the performance of each individual segment (segmentation).

Through the mechanism of consciously thinking about the desired movement, using the frontal cortex to regulate movement size or timing instead of the defective basal ganglia, PD patients arguably compensate for the neurotransmitter imbalance in the basal ganglia obtaining a more normal gait pattern [42]. The type of strategy, the frequency and duration of training should be considered according to disease severity, the capacity to learn, and whether there are coexisting conditions that limit the ability to practice (Table 1) [42].

\section{Assistive mobility devices}

PD patients with functional mobility impairments need to be able to move effortlessly, in a reasonable

Table 1

Strategies training guide adapted from Morris et al. (2010) [42]

\begin{tabular}{ll}
\hline & Training strategy: learning strategies to improve \\
& performance through practice \\
& Program: \\
& -3 times/week \\
Mild to moderate disease & - Peroids of 6 to 8 weeks (motor skill acquisition) \\
& - Burst of therapy 2 to 3 times/year (to promote \\
& retention of training)
\end{tabular}

Multi-tasking activities: use as training strategy, educate the patients on its risks 
amount of time throughout their day, accessing the same environments as others [45].

The use of assistive mobility devices (e.g., wheelchairs, walker) increases the ability of individuals to work, perform self-care, and engage in leisure and social activities independently, enhancing their functional performance, autonomy and participation [45-47].

Despite the potential advantages of assistive mobility devices, they are often underused or abandoned. The reason relates to a mismatch between a patient's functional needs, preferences and environmental constrains, and health professionals' perspectives. In 2017, Bettecken et al. [48] reported a relationship between PD patients' gait velocity using an assistive mobile device and their HrQoL. Surprisingly, the study did not show a relevant contribution of gait velocity to HrQoL. Also, a relevant portion of PD patients with high HrQoL preferred a low selfpreferred gait velocity to the use of an assistive mobile device. In a study to identify clinicians', patients' and caregivers' perspectives about relevant parameters and assessment tools for PD symptoms [49], Ferreira and colleagues reported that patients and caregivers have different perspectives when selecting the most relevant parameters for evaluating gait and sway domains. Patients and caregivers both highlighted the capability of performing ADL as the most important parameter. For clinicians, time consumed doing specific tasks was the most useful parameter.

\section{IMPLICATIONS FOR CLINICAL PRACTICE AND RESEARCH}

If $\mathrm{PD}$ patients are unable to move at an intensity and frequency that life requires, they may become excluded from social and occupational environments, which may negative impacts theirs global health status [7, 29].

Although the assessment of specific outcomes, like level of rigidity or intensity of tremor, is important, previous studies have shown that functional limitations, rather than physical impairments, were the most problematic aspect of a PD patient's disability profile $[5,7]$. The standard scale for evaluating impairments in PD is the MDS-UPDRS. However, besides being highly time-consuming, the objective evaluation of functional activities is limited. The TUG test is the most used tool to classify functional mobility and has been shown to be a valid predictor of performance in ADL. Yet, an exhaustive measurement system that adequately assesses functional mobility is still needed $[3,7,8]$.

More studies are needed to understand the relationship between the use of assistive mobility devices, PD patients' functional mobility and HrQoL. Perceived control may be the key aspect in explaining the intriguing conclusion that Bettecken and colleagues found in their study [48]. As mentioned above, perceived control is a powerful predictor of functioning and it seems that some patients place more value on the capability of performing ADL rather than the time it takes to perform specific tasks [26, 49]. We hypothesize that assistive mobility devices are acknowledge by patients as an effective solution only when perceived as a control gain. Otherwise, the use of assistive mobility devices is seen as a loss of autonomy with negative impact in $\mathrm{HrQoL}$ (even objectively improving gait characteristics such as velocity). It would also be interesting and useful to study if, for those PD patients who remain in employment, or who maintain an active social life, this hypothesis is valid.

\section{CONCLUSION}

Back to our initial question: is there a difference in the functional mobility of two PD patients with similar gait disturbance, one using an assistive mobility device, the other not? How do health professionals account for these differences?

This question can be seen from two different perspectives.

As a physiological ability, the two patients have the same degree of functional mobility, since what differentiated them was the use of an external device.

As an outcome measure eligible to be improved by a therapeutic intervention, the answer is not so clear. On one hand, assistive mobility devices enable a more active and safer lifestyle, allowing patients to continue to be engaged with their social and occupational environment. For this reason, the patient with an assistive mobility device has better functional mobility. On the other hand, this would only be true if the use of these devices increases patients' perceived control of their situation.

Understanding the determinants of functional mobility in individuals with $\mathrm{PD}$, such as the precedence of perceived control over an improved gait velocity, will help clinicians to more easily select the most appropriate therapeutic interventions based on 
an accurate, global, and personalized evaluation of patients' problems [7, 27, 31].

From this review on PD patients functional mobility, we highlight: 1) its benefits as a more global and functional outcome of patient assessment; 2) the important role of exercise programs, training strategies and assistive devices in improving patients' functionality and participation in social environments; and lastly, 3) the importance of taking into account patients' personal needs and wishes and environmental factors in order to optimize treatment strategies.

\section{ACKNOWLEDGMENTS}

No specific funding was received for this work.

\section{CONFLICTS OF INTEREST}

The authors have no conflict of interest to report.

\section{REFERENCES}

[1] Keus S, Munneke M, Graziano M, Paltamaa J, Pelosin E, Domingos J, Bruhlmann S, Ramaswamy B, Prins J, Struiksma C, Rochester L, Nieuwboer A, \& Bloem B, on behalf of the Guideline Development Group (2014) European Physiotherapy Guideline for Parkinson's Disease, 1st ed. The Netherlands.

[2] Lomax CL, Brown RG, \& Howard RJ (2004) Measuring disability in patients with neurodegenerative disease using the "Yesterday Interview." Int J Geriatr Psychiatry, 19, 10581064.

[3] Raggi A, Leonardi M, Ajovalasit D, Carella F, Soliveri P, Albanese A, \& Romito L (2011) Disability and profiles of functioning of patients with Parkinson's disease described with ICF classification. Int J Rehabil Res, 34, 141-150.

[4] Leonardi M, Meucci P, Ajovalasit D, Albanesi F, Cerniauskaite M, Invernizzi V, Lembo R, Quintas R, Sattin D, Carella F, Romito L, Soliveri P, Bussone G, D'amico D, Maggi L, Mantegazza R, \& Raggi A (2009) ICF in neurology: Functioning and disability in patients with migraine, myasthenia gravis and Parkinson's disease. Disabil Rehabil, 31, S88-S99.

[5] Leonardi M, Raggi A, Ajovalasit D, Bussone G, \& D' Amico D (2010) Functioning and disability in Parkinson's disease. Disabil Rehabil, 32(Suppl 1), S23-S32.

[6] van Uem JMT, Marinus J, Canning C, van Lummel R, Dodel R, Liepelt-Scarfone I, Berg D, Morris ME, \& Maetzler W (2016) Health-Related Quality of Life in patients with Parkinson's disease-A systematic review based on the ICF model. Neurosci Biobehav Rev, 61, 26-34.

[7] Forhan M, \& Gill SV (2013) Obesity, functional mobility and quality of life. Best Pract Res Clin Endocrinol Metab, 27, 129-137.

[8] Swarowsky A, Santos MP, Silva BA, Ovando AC, \& Ilha J (2016) Comparison between two functional mobility scales for Parkinson's disease directly applied to physical therapy practice: Cross-cultural adaptation and measurement properties. Eur J Phys Rehabil Med, in press.

[9] Burgess S, \& Rassmusson X (2016) Parkinson's narratives: Onset experiences and perceived benefits of preferred physical activity. Adv Soc Sci Res J, 3, 150-160.

[10] Lin SI, Lee HC, Chang KC, Yang YC, \& Tsauo JY (2017) Functional mobility and its contributing factors for older adults in different cities in Taiwan. J Formos Med Assoc, 116, $72-79$.

[11] King LA, \& Horak FB (2009) Delaying mobility disability in people with Parkinson disease using a sensorimotor agility exercise program. Phys Ther, 89, 384-393.

[12] World Health Organization (2002) Towards a common language for functioning, disability and health: The International Classification of Functioning, Disability and Health. Int Classif, 1149, 1-22.

[13] Duncan RP, \& Earhart GM (2014) Are the effects of community-based dance on Parkinson disease severity, balance, and functional mobility reduced with time? A 2-year prospective pilot study. J Altern Complement Med, 20, 757763.

[14] Magrinelli F, Picelli A, Tocco P, Federico A, Roncari L, Smania N, Zanette G, \& Tamburin S (2016) Pathophysiology of motor dysfunction in Parkinson's disease as the rationale for drug treatment and rehabilitation. Parkinsons Dis, 2016, 1-18.

[15] Kalia LV, \& Lang AE (2015) Parkinson's disease. Lancet, 6736, 1-17.

[16] Sveinbjornsdottir S (2016) The clinical symptoms of Parkinson's disease. J Neurochem, 139, 318-324.

[17] Raggi A, Leonardi M, Covelli V, Sattin D, Scaratti C, Schiavolin S, Willems M, \& Meucci P (2015) The ICF as a framework to collect and interpret data on the extent and variety of disability in neurological conditions. NeuroRehabilitation, 36, 17-22.

[18] Raggi A, Covelli V, Leonardi M, Meucci P, Scaratti C, Schiavolin S, Willems M, \& Sattin D (2015) Determinants of disability using count-based approaches to ICF-based definition of neurological disability. NeuroRehabilitation, 36, 23-29.

[19] Williams DR, \& Litvan I (2013) Parkinsonian syndromes. Contin Lifelong Learn Neurol, 19, 1189-1212.

[20] Postuma RB, Berg D, Stern M, Poewe W, Olanow CW, Oertel W, Obeso J, Marek K, Litvan I, Lang AE, Halliday G, Goetz CG, Gasser T, Dubois B, Chan P, Bloem BR, Adler $\mathrm{CH}$, \& Deuschl G (2015) MDS clinical diagnostic criteria for Parkinson's disease. Mov Disord, 30, 1591-1601.

[21] Robichaud JA, \& Corcos DM (2005) Motor deficits, exercise, and Parkinson's disease, Quest, 57, 79-101.

[22] Yoshii F, Moriya Y, Ohnuki T, Ryo M, \& Takahashi W (2016) Postural deformities in Parkinson's disease -Mutual relationships among neck flexion, fore-bent, knee-bent and lateral-bent angles and correlations with clinical predictors. J Clin Mov Disord, 3, 1.

[23] Merola A, Romagnolo A, Rosso M, Lopez-Castellanos JR, Wissel BD, Larkin S, Bernardini A, Zibetti M, Maule S, Lopiano L, \& Espay AJ (2016) Orthostatic hypotension in Parkinson's disease: Does it matter if asymptomatic? Parkinsonism Relat Disord, 33, 65-71.

[24] Boersma I, Jones J, Carter J, Bekelman D, \& Miyasaki JM (2016) Parkinson disease patient's perspectives on palliative care needs What are they telling us? Neurol Clin Pract, 6 , 209-219.

[25] Schilling BK, Karlage RE, LeDoux MS, Pfeiffer RF, Weiss LW, \& Falvo MJ (2009) Impaired leg extensor strength in 
individuals with Parkinson disease and relatedness to functional mobility. Park Relat Disord, 15, 776-780.

[26] McQuillen AD, Licht MH, \& Licht BG (2003) Contributions of disease severity and perceptions of primary and secondary control to the prediction of psychosocial adjustment to Parkinson's disease, Heal Psychol, 22, 504-512.

[27] Horak FB (2013) Objective biomarkers of balance and gait for Parkinson's disease using body worn sensors. Mov Disord, 28,1544-1551.

[28] Morris ME, Huxham F, McGinley J, Dodd K, \& Iansek R (2001) The biomechanics and motor control of gait in Parkinson disease. Clin Biomech, 16, 459-470.

[29] Mak MKY, \& Hui-Chan CWY (2002) Switching of movement direction is central to Parkinsonian bradykinesia in sit-to-stand. Mov Disord, 17, 1188-1195.

[30] Van Uem JMT, Walgaard S, Ainsworth E, Hasmann SE, Heger T, Nussbaum S, Hobert MA, Micó-Amigo EM, Van Lummel RC, Berg D, \& Maetzler W (2016) Quantitative timed-up-and-go parameters in relation to cognitive parameters and health- related quality of life in mild-to-moderate Parkinson's disease. PLoS One, 11, 1-15.

[31] Zijlstra A, Mancini M, Lindemann U, Chiari L, \& Zijlstra W (2012) Sit-stand and stand-sit transitions in older adults and patients with Parkinson's disease: Event detection based on motion sensors versus force plates. J Neuroeng Rehabil, 9, 75 .

[32] Louter M, van Sloun RJG, Pevernagie DAA, Arends JBAM, Cluitmans PJ, Bloem BR, \& Overeem S (2013) Subjectively impaired bed mobility in Parkinson disease affects sleep efficiency. Sleep Med, 14, 668-674.

[33] Koerts J, König M, Tucha L, \& Tucha O (2016) Working capacity of patients with Parkinson's disease - A systematic review. Parkinsonism Relat Disord, 27, 9-24.

[34] Wilcox SK (2010) Extending palliative care to patients with Parkinson's disease. Br J Hosp Med (London, Engl 2005), 71, 26-30.

[35] Poewe W (2006) The natural history of Parkinson's disease. J Neurol, 253, 2-6.

[36] Del Din S, Godfrey A, Mazzá C, Lord S, \& Rochester L (2016) Free-living monitoring of Parkinson's disease: Lessons from the field. Mov Disord, 31, 1293-1313.

[37] Karakaya MG, Bilgin SC, Ekici G, Köse N, \& Otman AS (2009) Functional mobility, depressive symptoms, level of independence, and quality of life of the elderly living at home and in the nursing home. J Am Med Dir Assoc, 10, 662-666

[38] Podsiadlo D, \& Richardson S (1991) The Timed "Up \& Go": A test of basic functional mobility for frail elderly persons. $J$ Am Geriatr Soc, 39, 142-148.

[39] Parashos SA, Elm J, Boyd JT, Chou KL, Dai L, Mari Z, Morgan JC, Sudarsky L, \& Wielinski CL (2015) Validation of an ambulatory capacity measure in Parkinson disease: A construct derived from the unified Parkinson's disease rating scale. J Parkinsons Dis, 5, 67-73.

[40] Bloem BR, Marinus J, Almeida Q, Dibble L, Nieuwboer A, Post B, Ruzicka E, Goetz C, Stebbins G, Martinez-Martin P, Schrag A, \& Movement Disorders Society Rating Scales Committee (2016) Measurement instruments to assess posture, gait, and balance in Parkinson's disease: Critique and recommendations. Mov Disord, 31, 1342-1355.

[41] Espay AJ, Bonato P, Nahab FB, Maetzler W, Dean JM, Klucken J, Eskofier BM, Merola A, Horak F, Lang AE, Reilmann R, Giuffrida J, Nieuwboer A, Horne M, Little MA, Litvan I, Simuni T, Dorsey ER, Burack MA, Kubota K, Kamondi A, Godinho C, Daneault JF, Mitsi G, Krinke L, Hausdorff JM, Bloem BR, Papapetropoulos S, \& Movement Disorders Society Task Force on Technology (2016) Technology in Parkinson disease: Challenges and opportunities. Mov Disord, 31, 1272-1282.

[42] Morris ME, Martin CL, \& Schenkman ML (2010) Striding out with Parkinson disease: Evidence-based physical therapy for gait disorders. Phys Ther, 90, 280-288.

[43] Behrman AL, Cauraugh JH, \& Light KE (2000) Practice as an intervention to improve speeded motor performance and motor learning in Parkinson's disease. J Neurol Sci, 174, 127-136.

[44] Canning CG, Ada L, \& Woodhouse E (2008) Multipletask walking training in people with mild to moderate Parkinson's disease: A pilot study. Clin Rehabil, 22, 226233.

[45] Meyer A (2009) Focus on pediatrics: Functional mobility vs. exercise. Directions, 3, 46-48.

[46] Auger C, Demers L, Gélinas I, Jutai J, Fuhrer MJ, \& DeRuyter F (2008) Powered mobility for middle-aged and older adults. Am J Phys Med Rehabil, 87, 666-680.

[47] Kumar A, Schmeler MR, Karmarkar AM, Collins DM, Cooper R, Cooper RA, Shin H, \& Holm MB (2013) Test-retest reliability of the functional mobility. assessment (FMA): A pilot study. Disabil Rehabil Assist Technol, 8, 213-219.

[48] Bettecken K, Bernhard F, Sartor J, Hobert M, Hofmann M, Gladow T, van Uem J, Liepelt-Scarfone I, \& Maetzler W (2017) Association of kinematic gait parameters with health-related quality of life in Parkinson's disease. PLoS One 12, e0176816.

[49] Ferreira JJ, Santos AT, Domingos J, Matthews H, Isaacs T, Duffen J, Al-Jawad A, Larsen F, Artur Serrano J, Weber P, Thoms A, Sollinger S, Graessner H, \& Maetzler W (2015) Clinical parameters and tools for home-based assessment of Parkinson's disease: Results from a delphi study. J Parkinsons Dis, 5, 281-290. 